\title{
Integration of Battle Damage Repair management in an Integrated Mission Management System
}

\author{
Lt Cdr F D Geertsma, MSc ${ }^{1}$ \\ Defence Materiel Organisation, Automation \& Integration, $N L$
}

\begin{abstract}
Synopsis
In the future, the Royal Netherlands Navy (RNLN) will face growing challenges at sea in an environment of ever increasing complexity, variety and speed. Ship systems will continue to evolve and become more interconnected. The ship's crew will be expected to do more with fewer numbers in less available time. To be able to meet these challenges the RNLN have started the Manning \& Automation roadmap to realize effective and integral support for operations on board.

A critical requirement for these steps to be made is the integration of information to enable automation to integrally support the crew in their operations. On current ships, information and automation of separate systems often are not integrated and survival relies on the crew connecting the dots of an ever-increasing complexity of data. Furthermore, during incidents crews rely on paper-based systems for Battle Damage Repair management to create an overview and support the ship's operations. These paper-based systems are slow, fault-sensitive, manpower-intensive and will potentially result in missed opportunities or critical errors, especially in a time-critical State 1 environment.

The RNLN have started several projects on the Manning \& Automation roadmap to develop integration and automation of the Internal and External battle into an Integrated Mission Management System. This paper will describe the current process and foreseen improvement for Battle Damage Repair management to support a more effective Internal and External Battle on ships of the RNLN with optimised crew numbers. It will demonstrate a large step in effectiveness and crew optimisation can be made by employing existing and emerging technology.
\end{abstract}

Keywords: Automation; Internal Battle; Integration; Battle Damage Repair management

\section{INTRODUCTION}

The environment in which warships are operating now and in the future is rapidly changing. Threats are constantly increasing in numbers, diversity and speed. Weapon systems are becoming ever more capable and intelligent. New technologies as Artificial Intelligence, UxV-systems and Big Data are quickly evolving, unlocking great threat-potential in an even more complex and time-critical environment. Furthermore, political situations are increasingly complex which results in an unknown status of potential threats with safe situations rapidly escalating into high threat environments. Warships and their crew therefore need to react and adapt faster to the quickly evolving and changing threats.

Fortunately, the systems on board are constantly evolving as well to enable warships to cope with these challenges. However, this results in increasing complexity and interdependency of these systems. The potential options and solutions to cope with external threats and internal incidents increase with exponential growth. This will further challenge the crew in a very time critical environment.

Currently, the manning trend is to reduce warship crew numbers due to an increasing shortage of specialised personnel, pressure on personnel budgets and a political ambition to submit as few personnel as possible to danger. This can result in overloading personnel, resulting in high-tech warships being operated in a less effective and slower manner. This can and probably will have critical consequences and could lead to missions failing, or even warships being destroyed.

\footnotetext{
${ }^{1}$ Authors' Biographies

Lt Cdr Frans Geertsma studied Marine Engineering at the Royal Netherlands Naval College and graduated with a Master's degree in Marine Engineering (electrical option) from the University College London. He is currently a systems engineer in survivability, automation and integration within the Defence Materiel Organisation. He has previously been employed as Marine Engineer Sea Riding Officer within FOST RN and been in charge of the Marine Engineering Department of HNLMS Evertsen. Earlier experience includes multiple sea and support appointments for the RNLN fleet.
} 
To cope with the abovementioned challenges, the RNLN have started the Manning \& Automation roadmap to realize effective and integral support for operations on board in order to cope with these growing challenges at sea, where fewer personnel will be expected to do more within less time.

\subsection{External Battle}

On current warships, automation already fulfils a critical role to enable the ship's crew to effectively utilize the available operational capabilities. However, situation dependent settings of these complex automated systems often need to be optimised by the crew. In the time-critical State 1 environment the crew often has insufficient time or knowledge to fully utilise the potential of their systems. Furthermore, management of the External Battle is still only partly supported by automation. Existing automation is primarily focussed on combining information only from different external sensors (as radars) and employed on system or sub-system level to ensure effective operation of these (sub)systems. This means the crew needs to process a lot of information in a very short time to enable them to decide which course of action is most effective. With an expanding number of possible options, this is a difficult task for the crew and potentially results in cognitive overloading of the crew. Therefore, often the most effective solution is not identified or, even worse, critical errors are made.

Technical developments in Sensor and Weapon systems, enhanced autonomy algorithms and information processing enable further automation support for management of the External Battle. However, if this is only done on a (sub)system level and for separate business processes, the full potential of the warship's systems and crew will not be utilized. One of the challenges is that the lower automation level of support systems (as propulsion and chilled water systems) limits the possibilities for further digital support of the External Battle. To enable future warships to become more effective and to allow the crew to use the full potential of these warships, a next step in automation needs to be taken with an integral view of the warship as a whole.

\subsection{Internal Battle}

An important requirement to conduct the External Battle is the availability of onboard systems and information from these systems. Availability needs to be maximised to ensure the full capabilities of the warship can be utilised. Furthermore, it is critical that Command is informed of the availability of these capabilities and on consequences of any defects and incidents. This information enables Command to assess which course of action is best for the warship and whether task handover or takeover within the taskforce is required to ensure a more global mission success.

Currently, the Internal Battle is only supported by limited automation and it relies heavily on manpower. The requirement to react faster means that loss and remaining availability of capabilities needs to be known by Command instantly, and furthermore, systems need to be safeguarded and recovered directly. To enable further support to the External Battle, availability of the ship's systems and their capabilities needs to be available digitally at a functional level.

\subsection{Paper structure}

This paper will focus on Battle Damage Repair Management (BDR-management) of the Internal Battle in State 1, although a similar analysis can be made for External Battle management and other readiness states. First, this paper will describe the current BDR-management process with its challenges. Thereafter, this paper will describe the envisioned steps for improvement of BDR-management to enable effective and integral support for warship business processes. This paper will finish with conclusions and recommendations.

\section{CURRENT BDR-MANAGEMENT OF THE INTERNAL BATTLE}

This section will describe current Battle Damage Repair-management (BDR-management) using the OODAloop decision cycle. The OODA-loop is the decision cycle of Observe, Orient, Decide, and Act, developed by military strategist and United States Air Force Colonel John Boyd [1]. The division of tasks between manning and automation will be visualised for all steps. The visualised division of tasks is based on expert opinion of operational ship's crew and FOST-training staff experience.

\subsection{Observe: Identification of suffered damage}

The first requirement after suffering damage is to build the damage picture to enable an estimation of the consequences of defects and incidents on the availability of ship's systems and their operational capabilities. This damage picture is generated from multiple sources: 
- Digital system information:

Most important systems are equipped with sensors to enable the crew to assess their availability. These information management systems generally are not connected and deliver information on different levels. For platform systems an Integrated Platform Management System (IPMS) collects and presents sensor information and alarms of platform system components. For sensor and weapon systems the Combat Management System (CMS) generates information on the technical and functional availability of combat (sub)systems. After suffering damage, the system information and alarms are evaluated by the ship's crew to assess which systems are damaged.

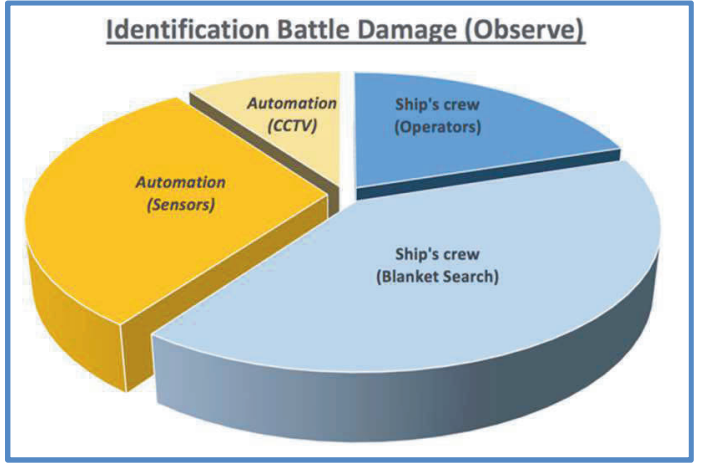

Figure 1. Decomposition of 'identification of suffered damage'

Furthermore, warships are equipped with sensors and CCTV to monitor the rooms on board for incidents such as fires and floodings (due to ship's hull leaks). This information is normally limited and will only give an indication that an incident might exist, but will not give much information on the state of the incident.

- Information from operators of the External Battle:

Operators will conduct mini-System Operability Checks (mini-SOC) to assess if systems are still performing properly. Combined with operational feedback from the use of the ship's systems, this will generate verbal or hand-written fault reports on the availability and performance of systems.

- Information from Blanket Search:

Unfortunately, the available information from the ship's information management systems is insufficient to ensure all defects and incidents are discovered and it contains insufficient detail to assess consequences on availability of capabilities and required recovery actions. Therefore, all available personnel assigned to the Internal Battle will conduct a Blanket Search to inspect all rooms and systems for damage and report their findings by voice. This Blanket Search will take approximately ten minutes to be fully conducted, dependent on the level of training and the extent of damage. On newer ships a smart Blanket Search is conducted where the crew decides to search only the damage area and its vicinity. However, this means the crew has to analyse all sensor alarms with information on the location of weapon impact and translate that to the expected damage area. This results in a delay of the start of the smart Blanket Search of minutes and a potential for making mistakes in deciding the expected damage area.

All information from abovementioned sources is collected by Defect Managers for system defects and incidents in Machinery Spaces and by leaders of the Fire and Repair Party Posts (FRPP I/C's) for incidents. They are bombarded by information and are required to constantly switch between tasks to capture all information, share their information and direct their personnel. The division of tasks is shown in Figure 1. It can clearly be seen that identification of suffered damage relies significantly on the ship's crew with some limited support by automation.

\subsection{Orient: Assessment of system and capability availability}

All separate sources of collected information need to be merged to ensure no defects or incidents are managed twice and all information is connected. Only within the information management systems, information is merged automatically and only at a limited scale and to different abstraction levels (e.g. subsystem level to functional level). The merging of separate sources of information for defects is managed on laminated paper cards (Figure 3) and for incidents due to their geographical nature on paper Incident Boards (Figure 4). Any information that needs to be shared with other persons, is communicated by voice over a communication line with high potential for mis-understandings.

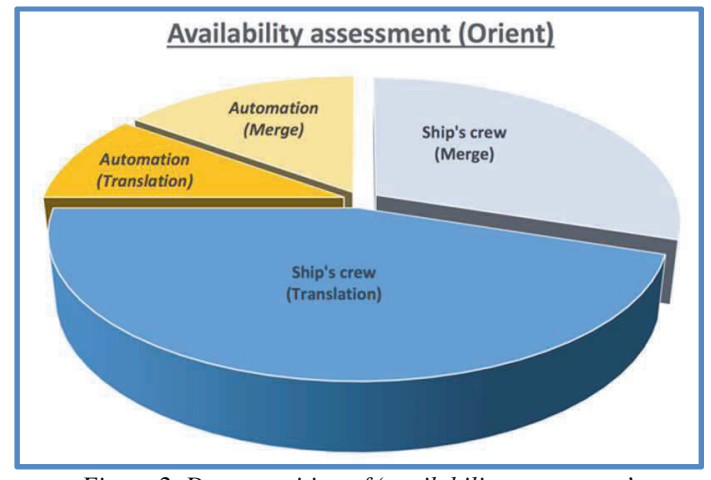

Figure 2. Decomposition of 'availability assessment' 


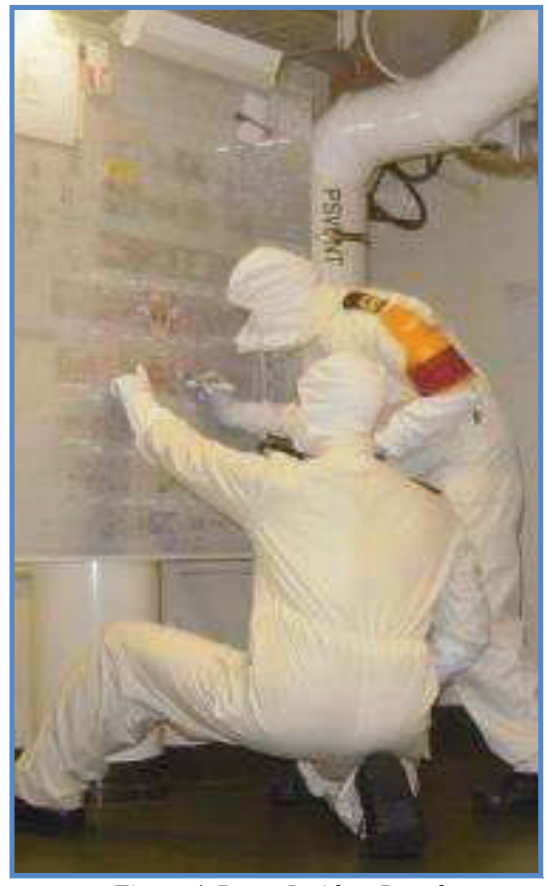

Figure 4. Paper Incident Board
The translation from observations to impact on availability of systems and capabilities relies on the experience and knowledge of the ship's crew with limited support by automation. In some cases, they are supported by paper aide memoires, paper Standard Operation Procedures (SOP's) and checklists. Directors in the Ship Control Centre receive the information by voice via a communication

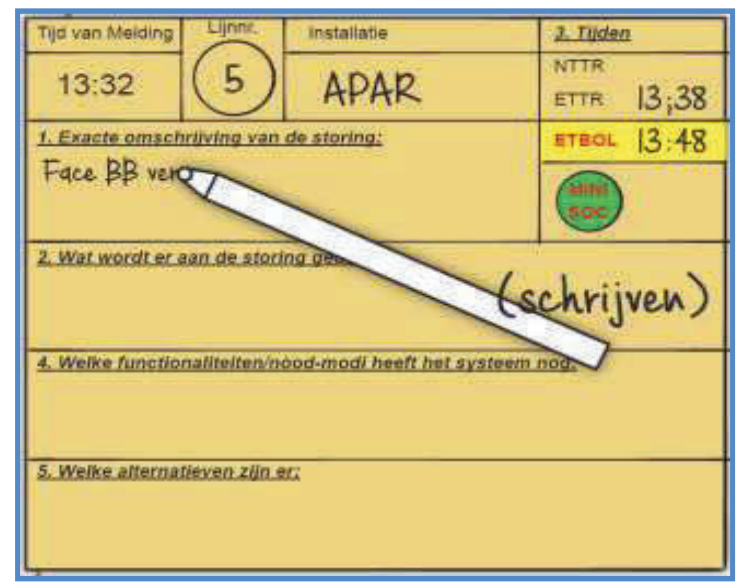

Figure 3. Paper defect cards line and use laminated paper cards to capture and translate this information. This process is labour-intensive and prone to errors. Because gathering data from the Defect Managers and FRPP I/C's takes up most time, little time is left to assess the consequences. The gathered information on system availability and remaining capabilities is compiled and communicated to Command by voice via communication lines, generally with large time-delays of 10 to 30 minutes.

The division of tasks is shown in Figure 2 and it is clear the process of availability assessment relies heavily on personnel with even less support by automation in processing these high amounts of data into information.

\subsection{Decide: Prioritisation and allocation of personnel}

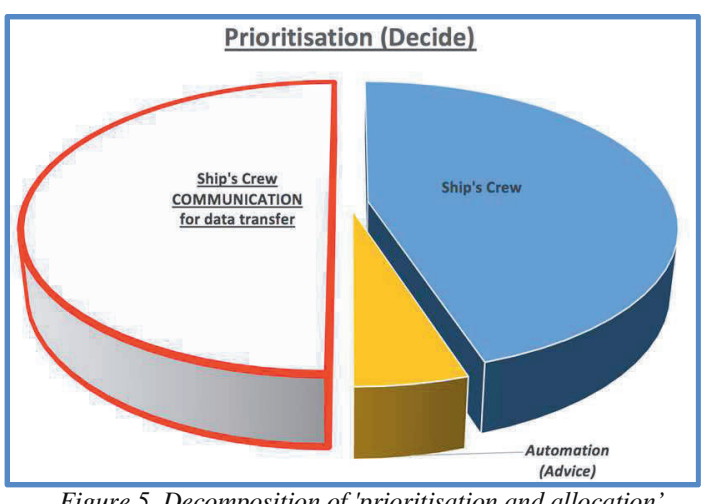

Figure 5. Decomposition of 'prioritisation and allocation'

With the impact of damage known, the importance to the mission is assessed. At sea, Command will always issue a Command Aim with the current mission focus. This gives sufficient information to assess which systems and capabilities are of high importance and need to be safeguarded or recovered first. The repair priorities will be established and aligned between Directors and communicated to Command for approval. Because the time and cognitive capacity of BDR-management is taken up mostly by communication and task-switching, little capacity is left for prioritisation and allocation of personnel. This results in potential for incorrect decisions. Furthermore, automation delivers only limited support. The reliance on ship's crew and shortage of time is visualised in Figure 5.

\subsection{Act: Recovery of capability}

To actually safeguard and restore capabilities, personnel will be tasked to conduct repairs or fight incidents in line with the established priorities. This is done by voice and captured on the same laminated paper cards by Defect Managers and FRPP I/C's.

Critical systems on board warships have been designed with various redundancy measures to ensure sufficient survivability during battle damage. To utilize the available redundancy manual reconfigurations are required, further loading a limited crew and delaying the restoration of capabilities. In some cases, the actual defect needs to be located during the Blanket Search before reconfigurations can be started. This sometimes has critical consequences, as for example Chilled Water systems (vital for sensor and weapon systems) will most probably be drained of fluids before the defect is located. [5]

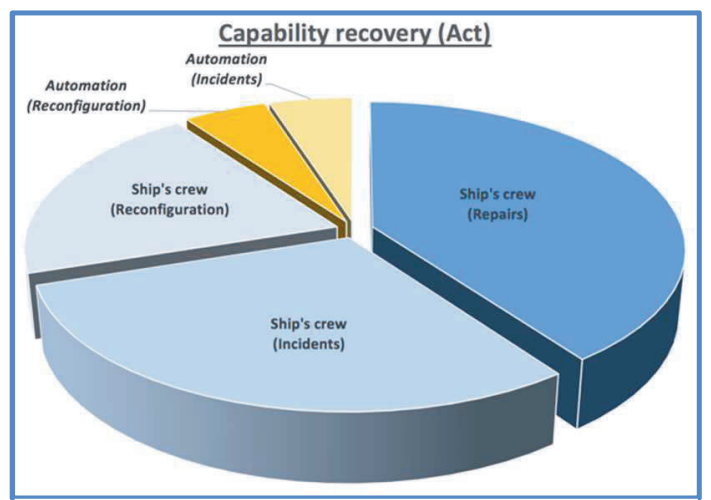

Figure 6. Decomposition of 'Recovery of capability'

Most actions to fight incidents and repair systems are manpower intensive and time-consuming. Some automation is employed to assist in fighting incidents, but large teams are still required. The division of tasks is shown in Figure 6. 


\subsection{Conclusion}

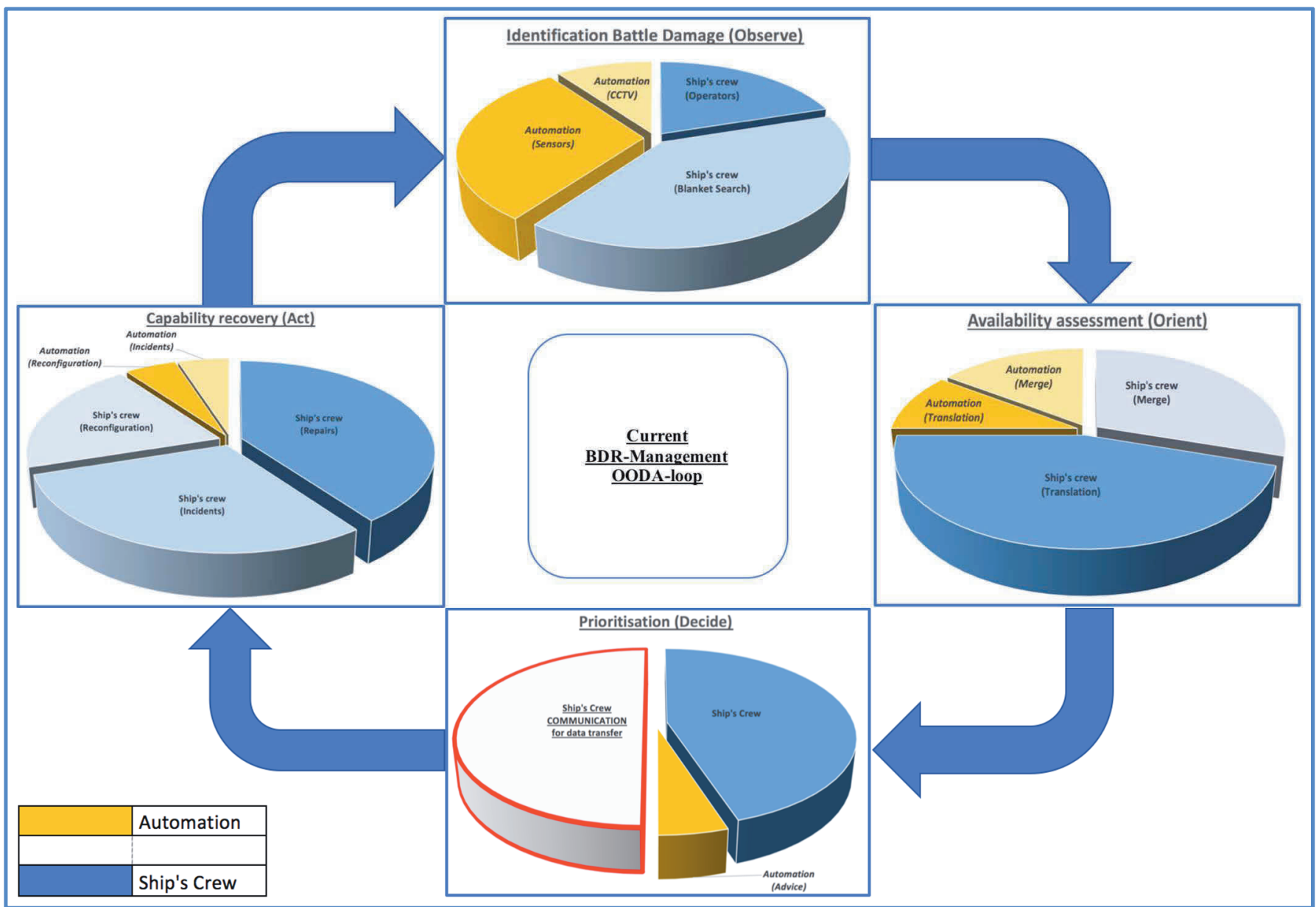

Figure 7. Current BDR-Management OODA-loop

The current BDR-management process is highly dependent on voice-communication and, as shown in Figure 7, all steps of the OODA-loop are primarily dependent on the ship's crew (blue sections) with limited support by automation (orange sections). Personnel is the continuous linking pin to connect and share information, and to connect the separate steps of the OODA-loop. The high burden of tasks and frequent task-switching, combined with a hectic environment puts a high cognitive load on the ship's crew with potential to overload personnel.

Consequently, Command is informed late of availability of systems and capabilities, and recovery of capabilities is slow with a high manpower requirement. This will result in Command making sub-optimal decisions (internally or externally) with potential critical consequences for the ship or taskforce. This is often experienced by ship's crews during operational training where (simulated) Battle Damage is suffered. Significant training is required to ensure the ship's crew is capable in BDR-management, and even with a trained crew the full potential of the warships will be limited by this slow and fault-sensitive process.

Furthermore, availability of systems and operational capabilities is only available on paper and therefore not digitally available which limits further automated support for Internal and External Battle business processes.

\section{BDR-MANAGEMENT IMPROVEMENT}

To improve the Internal and External Battle business processes, BDR-management needs to become more effective and information needs to be available digitally. Currently ship's crew are tasked with all steps of the OODA-loop and required to connect all information manually. Humans are generally not effective at processing large quantities of information and conducting repetitive or procedural steps. These tasks can be executed more effectively with automation and mechanisation. This will free up time for the strong human attributes: decisionmaking with incomplete information and task-execution in complex environments.

This section discusses the steps towards a more effective BDR-management. Again, the OODA-loop is used to visualise the division of tasks. First an IT-architecture is described to support automation of Internal Battle business processes. 


\subsection{Integral ship-wide IT-architecture}

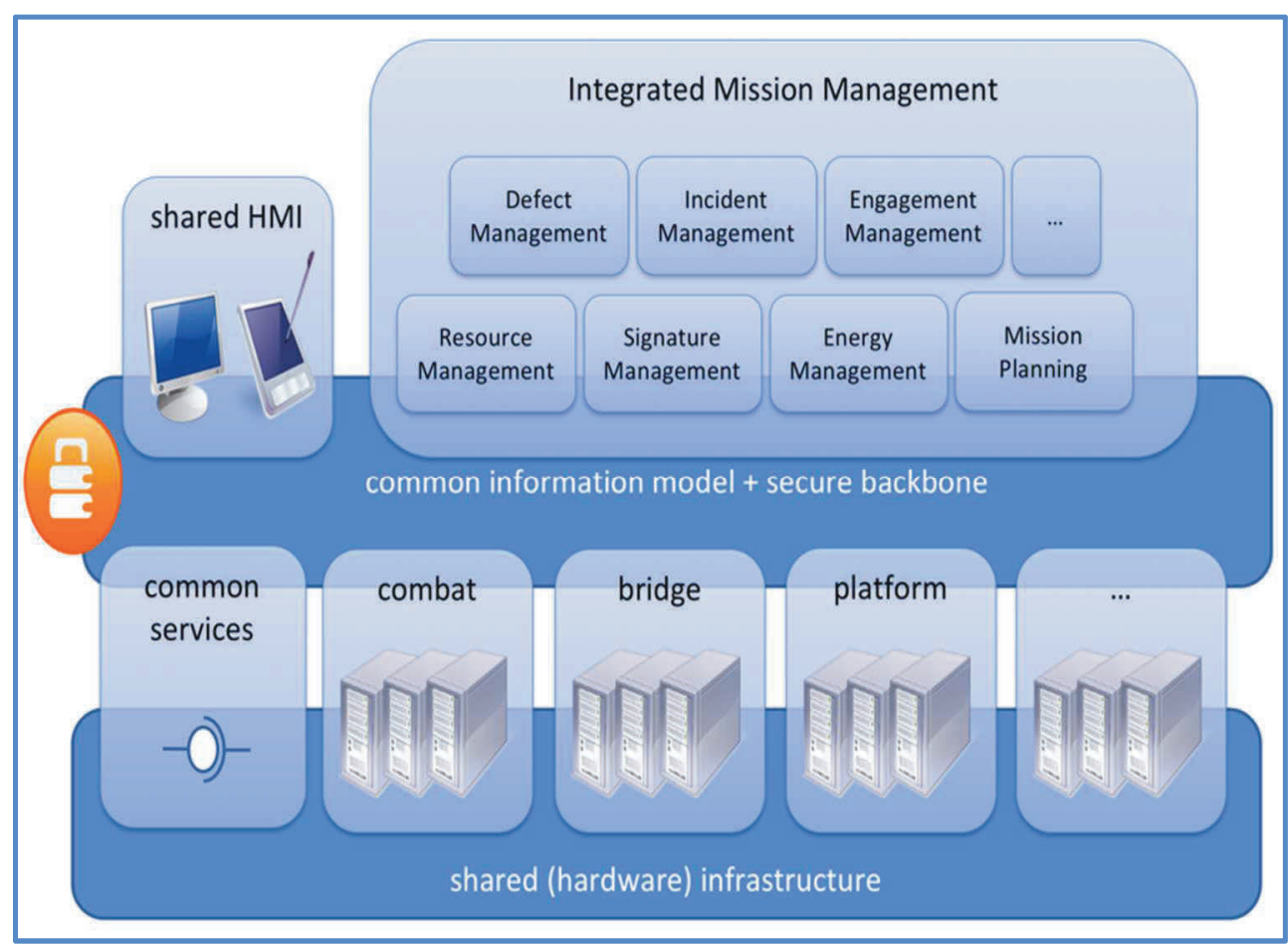

Figure 8. GAUDI common IT-architecture solution [2]
For the envisioned improvements, it is critical that all information can be combined in an effective and efficient manner. This requires an integrated and secure ITarchitecture solution and combined information model(s). Existing IT-systems (stovepipes as CMS and IPMS) have to be kept intact, as it is simply too costly to develop them from scratch. Therefore, the RNLN has started

a project with industrial partners to develop a flexible IT-architecture (GAUDI) to disclose the necessary information from and for the stovepipes in a systems-of-systems approach (Figure 8). The envisioned integral applications can then be developed which can utilize all available information. These applications will be placed in an Integrated Mission Management System (IMMS), which will allow integral support to both the External and Internal Battle, including integration of offboard systems and UxV's. This will remove the requirement for ship's crew to combine information from separate systems manually and enable further integral support by automation [2]. This IT-architecture therefore will enable effective BDR-management to be developed.

\subsection{Observe: Increased information availability for damage overview}

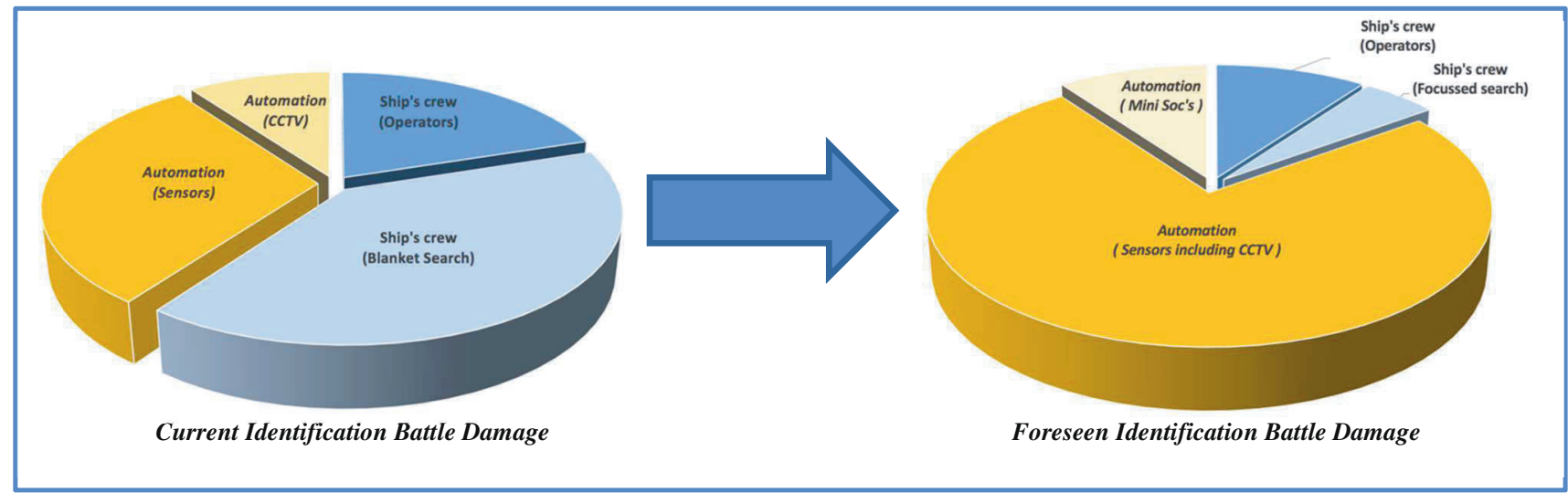

Figure 9. Foreseen Identification of Battle Damage

Currently systems are fitted with insufficient sensors and not all sensor information of sub-systems is available in information management systems. Therefore, systems will need to be fitted with more sensors and more evolved diagnostics on system and functional level. Furthermore, use of machine learning and predictive maintenance techniques will enable the information management systems to deliver information of a higher quality enabling the ship's crew to make decisions instead of processing information. The mini-SOC-process can mostly be automated and the results combined with sensor information. The RNLN is starting studies to decide which information is required and how this information can be processed to provide a better overview of system statuses. 
Unfortunately, it is unrealistic to expect all Commercially-Of-The-Shelf (COTS) systems to be fitted with sufficient sensors and diagnostics, although the advent of Internet Of Things (IOT) will facilitate this development. Furthermore, systems can also be impacted by incidents in the rooms they are located. Therefore, is it essential that rooms are also fitted with sensors to enable automation to inform the ship's crew of the state and integrity of rooms and to enhance the information of sensors on systems. Sensor costs are rapidly reducing and techniques to combine information of multiple sensors are also developing rapidly. By combining these sensors with CCTV and imaging processing software the status of rooms and its systems can be monitored.

By combining room and system information, a complete status overview can be generated and the requirement for a Blanket Search of the ship removed, with the exception of those rooms where the sensors have been significantly damaged. This will enable the ship's crew to immediately direct the repair and damage control teams to the high priority defects and incidents to safeguard and restore capability.

Implementation of these developments will result in the burden for identifying the suffered Battle Damage to be primarily taken over by automation with only a small requirement for ship's crew interaction as shown in Figure 9. The author expects this benefit to significantly outweigh the challenges of development of sensor fusion algorithms and implementation of adapted BDR-procedures.

\subsection{Orient: Information processing for system and capability overview}

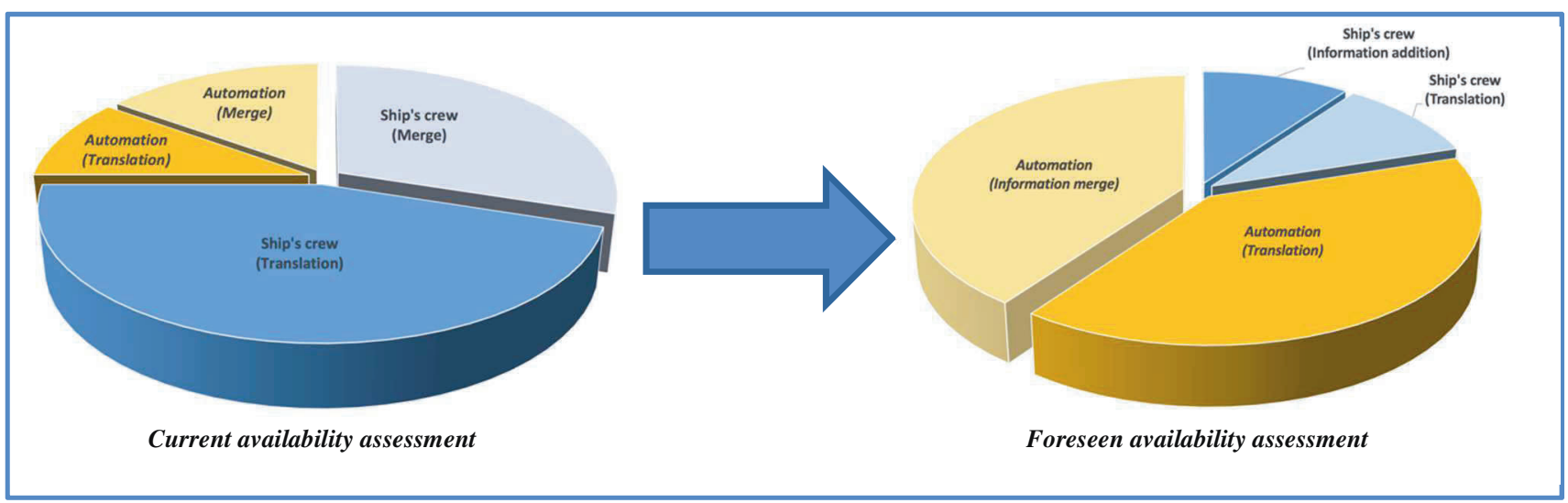

Figure 10. Foreseen system and capability assessment

\subsubsection{Information processing into a common information structure}

Information needs to be available on the same functional level and in the same information structure to enable automation to merge and process information. For most business processes resource management of systems and capabilities is required to allow an integral approach for the Internal and External Battle. The RNLN therefore has started several projects to aggregate information to the functional level of resources:

- Development of automation within IPMS to create hierarchy for unconnected component sensor information to deliver system status information [3];

- Investigation into frameworks to enable separate management applications within the IMMS to work together, identifying and potentially resolving any conflicts;

- Digitisation of information of the status and capabilities of personnel;

- Digitisation of the Command Aim.

This will enable all resources on board (systems, personnel and operational capabilities) and their availability to be managed integrally.

\subsubsection{Information digitisation and information addition by ship's crew}

With an integrated and digital information overview, the workload for the ship's crew to capture and distribute this information will be minimised. However, the ship's crew will still need to add human observations, inspection results and progress of repairs. Therefore, the RNLN has started projects to replace paper methods to capture information in order to enable ship's crew to combine digital and human observations (section 3.3.2.1 and 3.3.2.2). One of the major lessons learned is that the Human Machine Interface (HMI) of these applications is critical for the use and performance. Time delay due to the digital capture of information in comparison with the manual paper process will not be accepted by operators. The resulting HMI-concepts are expected to be effective for other business processes as well. 


\subsubsection{Electronic Incident Board}

For Damage Control incidents a geographical overview is required. The RNLN has developed an Electronic Incident Board (EIB) with RH Marine to replace the paper Incident Boards (Figure 11). The majority of the RNLN-fleet has now been fitted with EIB's. The experiences with these boards have been extremely positive during operational periods and training at FOST with feedback that EIB is effective and instinctive, ensuring the damage picture at separate nodes is identical and allowing the Damage Control Officer to focus more on Command and Control [4]. The incident picture is shared instantaneously without the requirement for voice communication, preventing misaligned pictures and delays. EIB is being further developed to combine separate plot data into the structure of incidents. This will enable incidents and SOP's to be managed digitally and consequences of incidents to be assessed digitally.

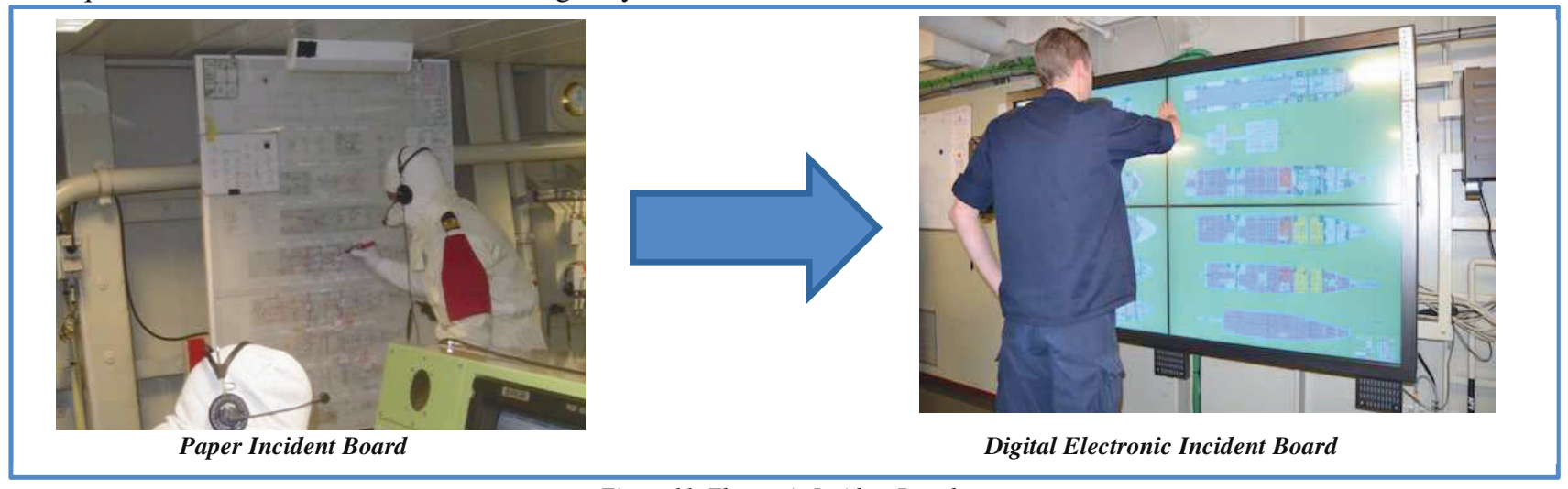

Figure 11. Electronic Incident Board

Further developments are started to support Damage Control business processes with more automation, as for example smoke boundary management, boundary cooling management and electrical isolations. In the future it is foreseen this application will also be made fit for geographical views on systems and their defects. This will enable a geographical and detailed view of all defect and incidents, ensuring the relations and conflicts can be interpreted. [3]

\subsubsection{Digital BDR-tool}

For defects, the RNLN has started a project with TNO to develop a digital BDR-management tool to replace the manual paper process (Figure 12). In this tool all management levels will have their own view with information on the required abstraction level. As the information model will ensure all defects are linked to systems and capabilities, a system and capability overview can be generated automatically (Figure 13).

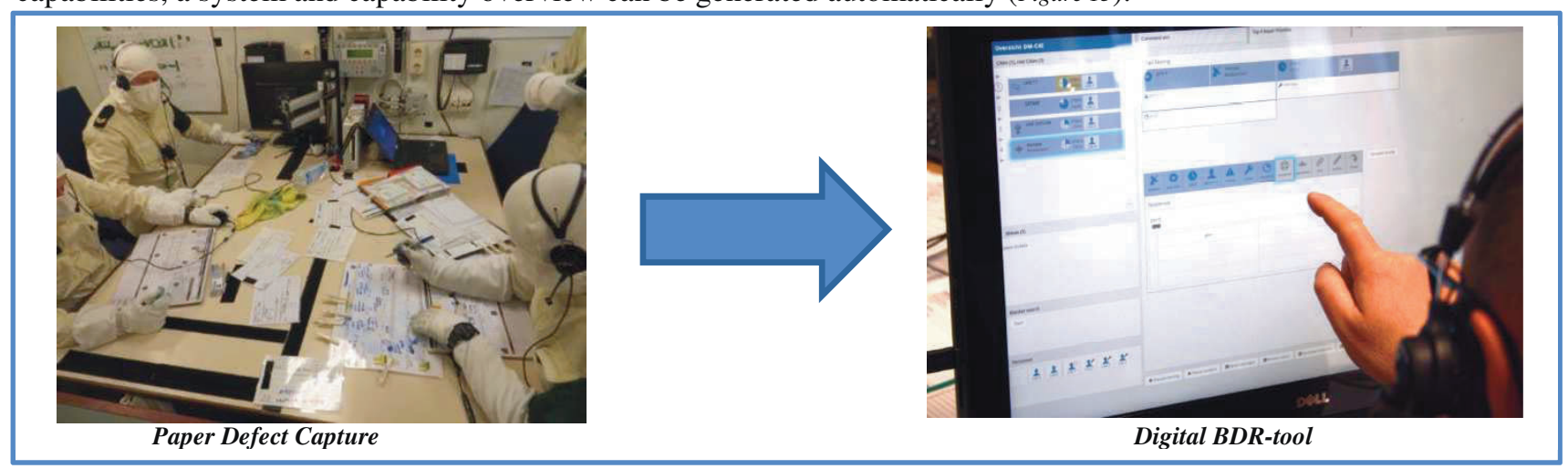

Figure 12. Digital BDR-tool

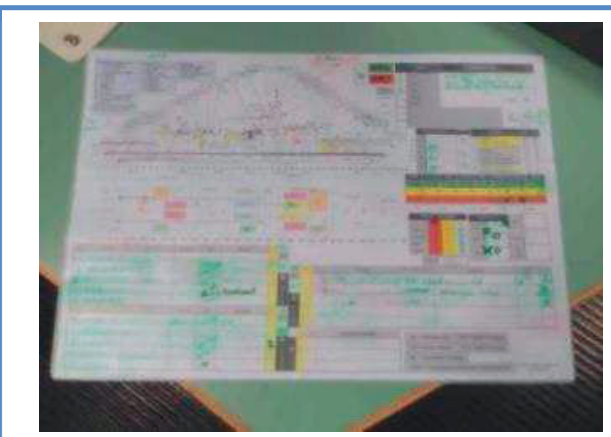

Paper defect and capability overview

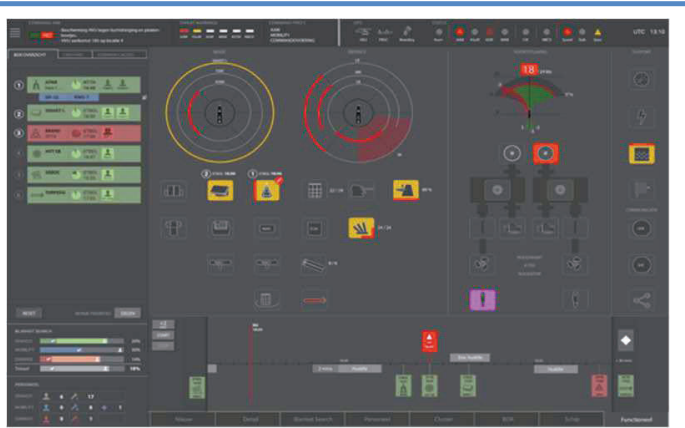

Digital capability overview

Figure 13. Digital ship's capability overview 
A prototype BDR-tool has been developed and tested with an operational ship's BDR-management team undergoing a standard Battle Damage training scenario. Feedback of the ship's crew was that the damage overview with the BDR-tool is much clearer with near real time information

distribution, resulting in Command being constantly aware of the discovered damage and resulting capability loss. A project is now started to develop the prototype for production and installation on board. At first the tool will have limited integration with other systems. This only partly relieves the burden to capture all information of Defect Managers and therefore the information capture delay and potential for faults is expected to only be partly reduced. The requirement to share information by voice on the other hand will be minimised, ensuring a shared picture and reduction in potential faults and delay in relaying information. When the information from the information management systems is at the required functional level, this information will be incorporated as well to combine digital information of sensors and human observations. This will further reduce the workload of the

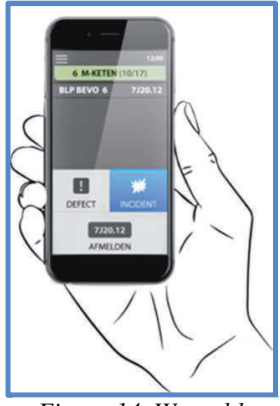

Figure 14. Wearable BDR-tool ship's crew and eliminate delays and potential faults. For future ships, methods are investigated to support repair personnel with a wearable device in order to supply them with the information they need and to enable them to add information digitally, further relieving Defect Managers and FRPP I/C's (Figure 14).

\subsection{Decide: Increased time and automation support for prioritisation and allocation}

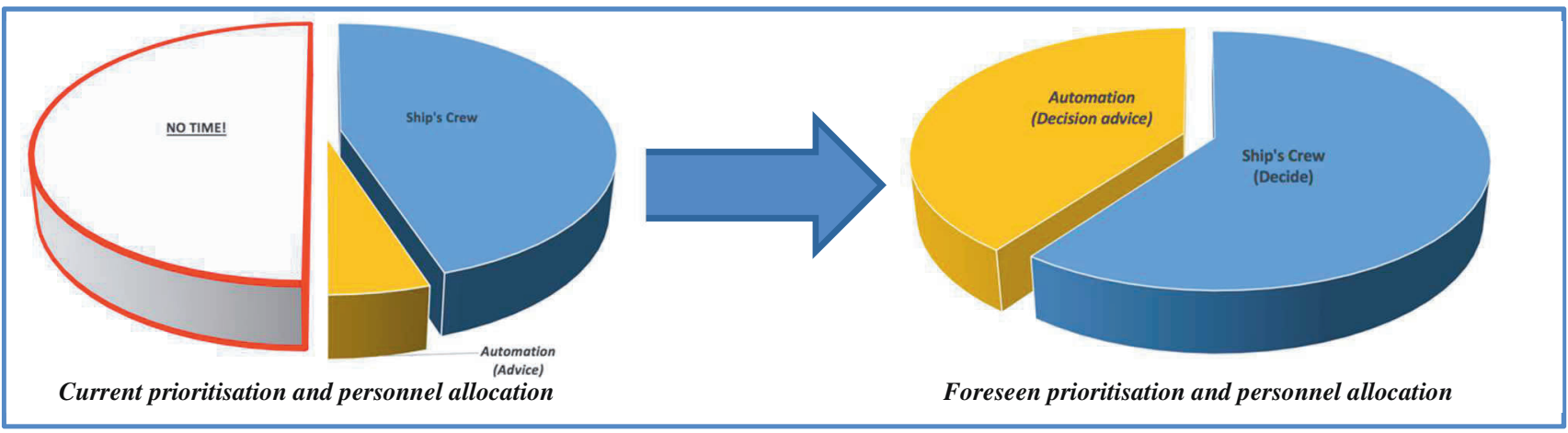

Figure 15. Foreseen Prioritisation and personnel allocation

The aforementioned improvements will result in less time lost in the steps Observe and Orient and will supply the ship's crew with more time and cognitive capacity to align between managers and prioritise defects and incidents. Furthermore, when all resource-information (systems and personnel), BDR-information, Command Aim and required External Battle resources are digitally available, it is possible to develop automation to generate advice for prioritisation and personnel allocation. This will free up more time to enhance quality, cope with unknown situations and ensure the most effective solution is selected to safeguard and recover capability, as visualised in Figure 15.

\subsection{Act: Enhanced automation and mechanisation}

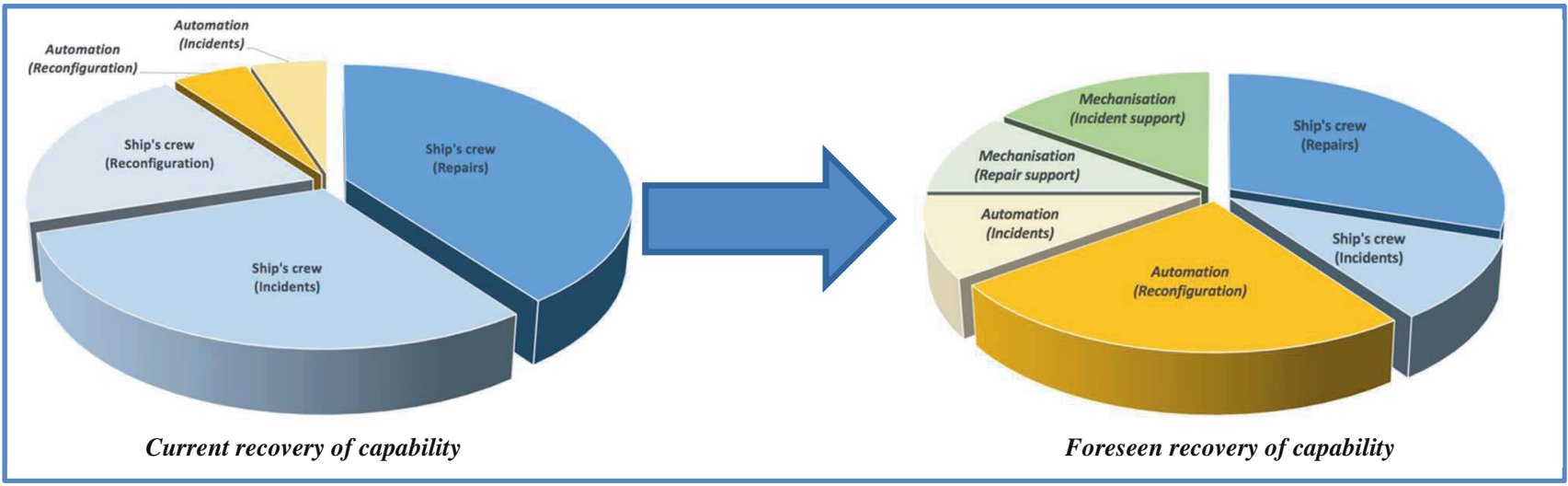

Figure 16. Foreseen capability safeguard and recovery

Due to a real-time generated defect and incident picture, personnel can be tasked directly to safeguard and recover capabilities. Technology progression enables repairs and recovery from incidents also to be supported. Especially for the reconfiguration of systems with inherent redundancy, automation and mechanisation deliver the potential for increased reaction time and lower manpower requirements. Together this will result in capability to be maximised directly and automatically after suffering damage. This will enable available personnel to be directly tasked to fight incidents and repair defects and furthermore to conduct their tasks more effective. The support of automation will deliver more capacity for the ship's crew to recover capabilities faster, as visualised in Figure 16. 
RNLN started several projects to automate and mechanise actions required to limit the impact of defects and incidents and to automatically reconfigure systems to utilize the available redundancy (sections 3.5.1, 3.5.2 and 3.5.3).

\subsubsection{Automated Decision System for IPMS}

The Automated Decision System (ADS) automatically conducts IPMS-actions to isolate and reconfigure after defects and then advices the ship's crew on the actions to take. This application has been implemented on the Dutch OPV-class early 2018 and is being further developed to assist in electrical isolation of rooms when incidents like fires and floods occur [3].

\subsubsection{DINCS}

DINCS is a software agent-based automation system for distributed platform support systems on warships, such as chilled water. DINCS autonomously detects and isolates defects, reconfigures systems to circumvent the defect and manages demand and supply. DINCS utilises the available redundancy and exploits alternative pathways in case of damage. Timely isolation and reconfiguration will be conducted with minimal operator error due to the increased autonomy, preventing loss of capabilities due to delayed human reaction time and resulting in a maximized fight-through capability. [5]

\subsubsection{Mechanisation}

Furthermore, manpower intensive tasks are investigated to find other manpower-lean solutions for coping with these incidents and defects. For fires one of the solutions seems to be the use of high pressure watermist to ensure fires are automatically extinguished or at least contained to a small fire. The newer RNLN-classes are fitted with high pressure watermist in all spaces.

\subsection{Conclusion}

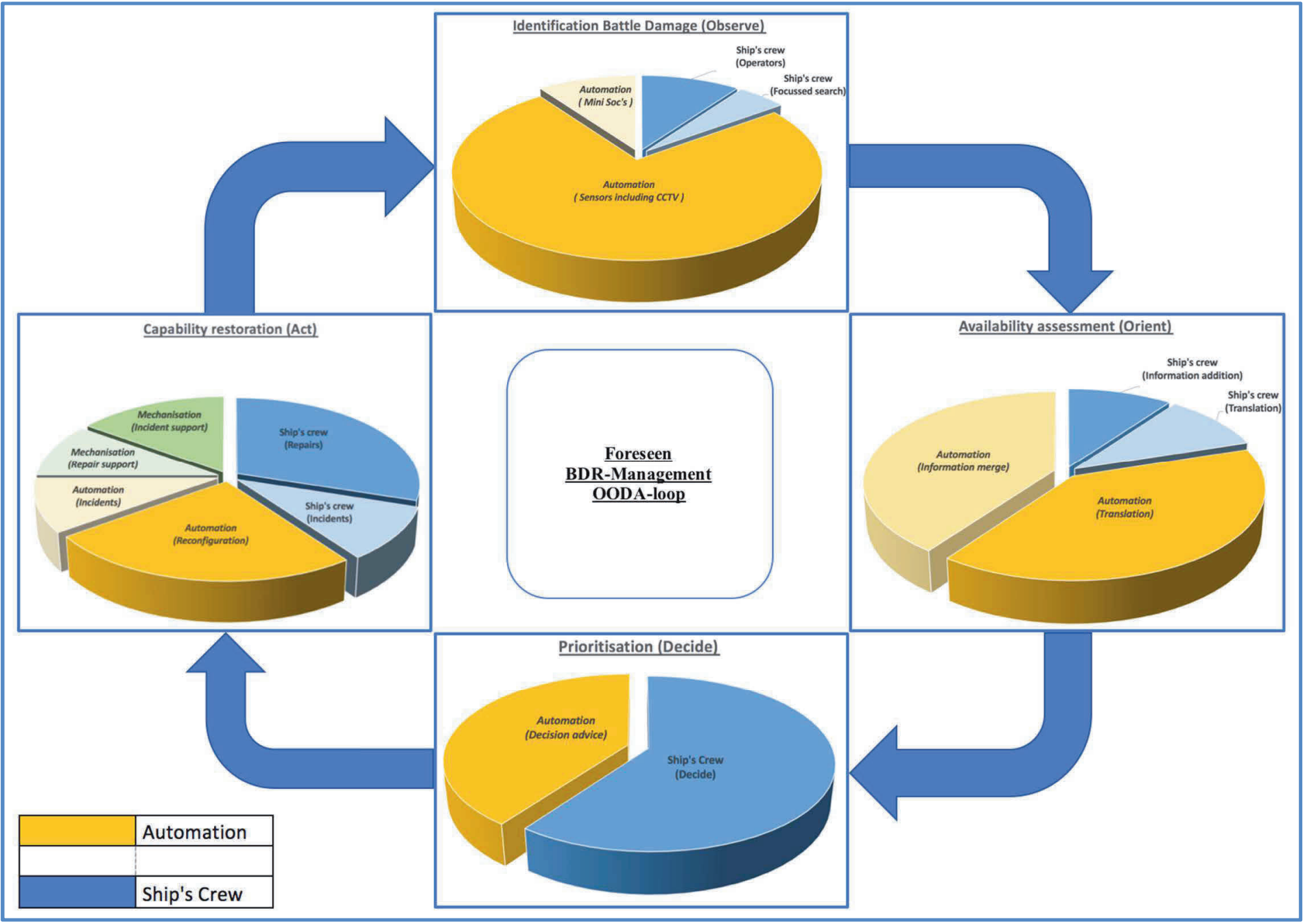

Figure 17. Foreseen BDR-Management OODA-loop 
By applying automation (orange sections) to BDR-management the Observe and Orient burden for Ship's crew (blue sections) can be minimised. This will free up time and cognitive capacity for Decide and Act processes. By also applying automation to support Decide and Act, more capacity will be available to fight incidents and conduct repairs. Therefore, capabilities will be regained faster. The shift from ship's crew capacity from Observe and Orient to Decide and Act is visualized in Figure 17. Furthermore, by applying automation, the internal status of the ship will be available for automation to further support the External Battle.

It is essential for implementation of automation to take the whole business process of the warship into account. Although automating parts of the process can result in improvements and it allows a step-by-step, clear overseeable development, there is a chance of creating an automation gap: the introduction of more workload during introduction of automation to a part of the process, as the ship's crew have to manually interface between separate processes (or steps of the OODA-loop). This has to be prevented and could result in the delay of the introduction of automation to a part of the process until the larger process or more processes together can be automated.

In October 2017 the potential of information integration of GAUDI and integral automation has been demonstrated during a Manning \& Automation symposium with a GAUDI-demonstrator with interaction between IPMS, CMS, Integrated Bridge Management System (IBMS), Electronic Incident Board (3.3.2.1), BDR-tool (3.3.2.1), DINCS and an External Battle engagement manager. Information of damage from digital systems, human observations and automated repair solutions (DINCS) was integrated automatically and distributed directly. This resulted in a near real-time integral system availability overview for External Battle applications. The engagement manager therefore could support the operator with engagement solutions adapted to the changing system availability due to suffered damage. Interacting with the IBMS enabled quicker ship reaction to threats. The symposium demonstrated a significant reduction of ship's crew workload, a crucial improvement of reaction time for Internal and External Battle, and it highlighted the further potential of these developments.

\section{CONCLUSIONS}

This paper has first described the current BDR-management process and then highlighted potential improvements (Figure 18).

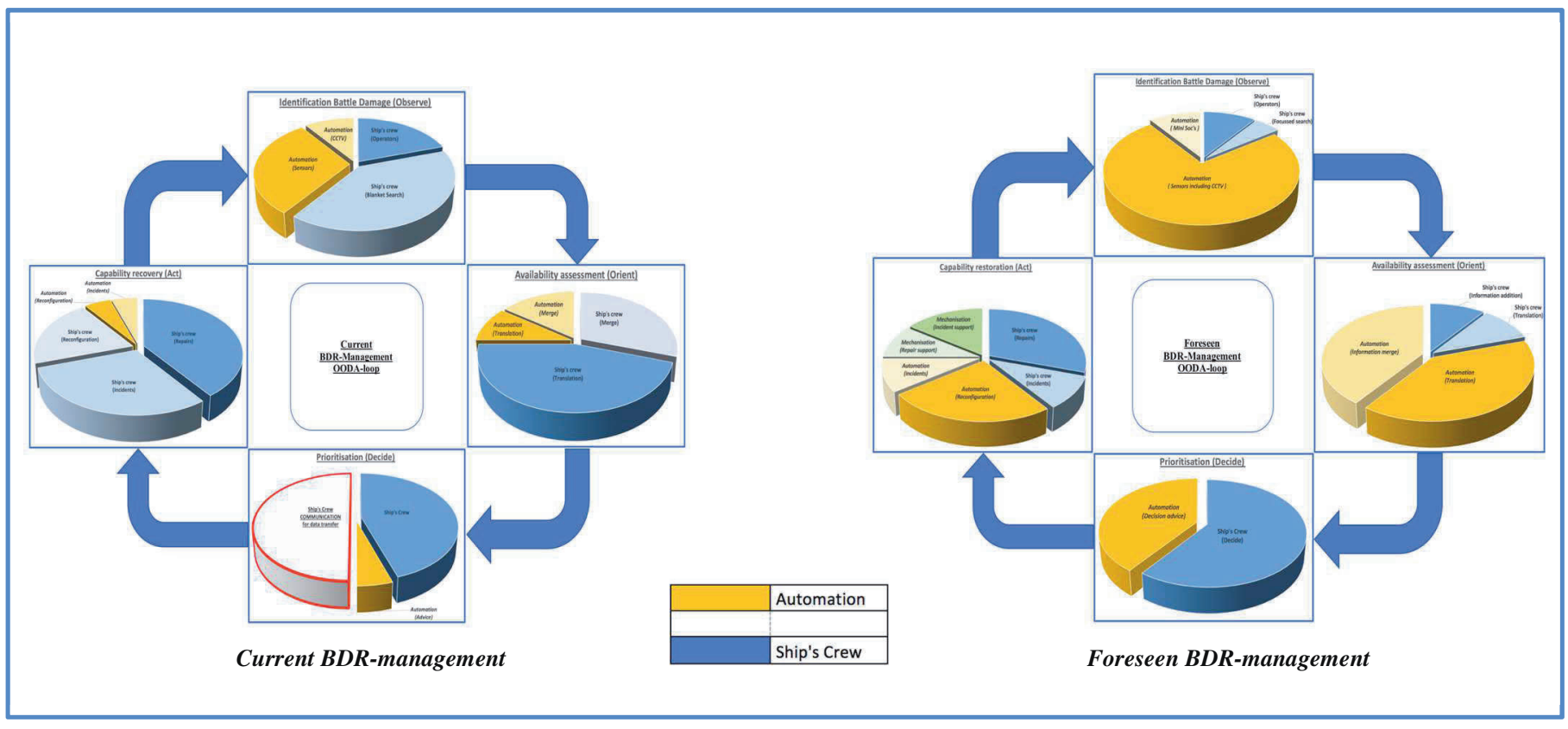

Figure 18. Comparison of current and foreseen BDR-management

The author concludes:

- Although current BDR-management is robust and flexible, it is slow, manpower-intensive and faultsensitive due to a high reliance on voice communication and an overloaded ship's crew.

- BDR-management is crucial to conducting missions successfully and it needs to improve to enable further support of the External Battle.

- It is crucial to ensure ship-wide information integration and availability. Therefore, the IT-architecture needs to support this and common information models are required. 
- The largest improvement can be made by automating the Observe and Orient steps of the OODA-loop, i.e. the identification of Battle damage and translation into system and capability availability. This will ensure a digital real-time overview of the availability of ship systems and remaining capabilities. Furthermore, this will reduce overloading ship's crew significantly and enable crew optimisation.

- If the Observe and Orient steps of the OODA-loop are automated and all information integrated, more time will be available for the Decide step of the OODA-loop, the OODA-loop can be executed quicker and further improvement can be gained by automation to support this Decide step, i.e. prioritisation and allocation of personnel.

- Ship capabilities can be safeguarded and regained more swiftly by automating and mechanising manpower intensive tasks of the Act step of the OODA-loop, i.e. reconfiguration, repairs and control of incidents. This will potentially also enable further crew optimisation.

\section{RECOMMENDATIONS}

This paper focussed on the Internal Battle BDR-management in State 1, but a similar analysis can be done for the External Battle, other readiness states, all operational and support business process on board or ashore. These also cope with the same challenges and have massive potential for improvement. Therefore, the author recommends:

- to do the same analysis for the External Battle, other readiness states and all business processes of warships onboard and ashore;

- $\quad$ to develop the mentioned technologies and applications for all other readiness states, because:

○ the same challenge to operate warships during other readiness states exists;

$\circ \quad$ use of the technologies and applications during other readiness states will ensure competence during Battle or Incidents.

- to conduct a study on how to effectively manage ship data to ensure the configuration, maintenance and programming effort to configure and maintain all applications is reduced to a minimum. The author expects that in order to enable continuous cost-effective support to warships, it is critical that all support and technical information is combined and only one master dataset exists, which can be used by all relevant suppliers.

\section{ACKNOWLEDGEMENTS}

This paper is the result of and would not have been possible without the work and vision of many people within RNLN (MoD, CZSK, and JIVC), RH Marine, Thales and TNO. The author would like to express his appreciation for the effort of these persons.

\section{REFERENCES}

[1] M. Revay and M. Liska, 'OODA loop in Command \& Control Systems'.

[2] J. Skowronek, M. Ditzel, CDR J.C. Schreurs, L. Franke and P. Rozendaal, 'GAUDI: innovative architecture for integrated maritime mission systems in support of advanced optimised manning', proceedings INEC 2016.

[3] F.D. Geertsma, N.A. Badon Ghijben, E.J. Middeldorp, R. Wouters, 'Adaptive automation to maximise warship capabilities during incidents', proceedings INEC 2016.

[4] R.D. Geertsma and N.A. Badon Ghijben, 'Experiences on implementation of electronic incident boards in the Royal Netherlands Navy', proceedings INEC 2014.

[5] J.A.A.J. Janssen, J. Butler, P. Worthington, F.D. Geertsma and M. den Hartog, 'Autonomous, adaptive, aware: DINCS', proceedings INEC 2016.

[6] E.J. Middeldorp, N.A. Badon Ghijben and R.D. Geertsma, 'Advanced PMS functionality in the Royal Netherlands Navy: automation to support the internal battle', proceedings INEC 2012.

[7] R.D. Geertsma, N.A. Badon Ghijben and O.R. Zwart, 'Fire Fighting and Damage Control: enabling future crew reduction', proceedings Engine As A Weapon 2013. 